\title{
Are H I Supershells the Remnants of Gamma-Ray Bursts?
}

\section{Citation}

Loeb, Abraham, and Rosalba Perna. 1998. "Are H [CSC]i[/CSC] Supershells the Remnants of Gamma-Ray Bursts?” The Astrophysical Journal 503 (1): L35-37. https://doi.org/10.1086/311536.

\section{Permanent link}

http://nrs.harvard.edu/urn-3:HUL.InstRepos:41393208

\section{Terms of Use}

This article was downloaded from Harvard University's DASH repository, and is made available under the terms and conditions applicable to Other Posted Material, as set forth at http:// nrs.harvard.edu/urn-3:HUL.InstRepos:dash.current.terms-of-use\#LAA

\section{Share Your Story}

The Harvard community has made this article openly available.

Please share how this access benefits you. Submit a story.

\section{Accessibility}




\title{
ARE H I SUPERSHELLS THE REMNANTS OF GAMMA-RAY BURSTS?
}

\author{
Abraham Loeb and Rosalba Perna \\ Harvard-Smithsonian Center for Astrophysics, 60 Garden Street, Cambridge, MA 02138 \\ Received 1998 June 9; accepted 1998 June 11; published 1998 July 17
}

\begin{abstract}
Gamma-ray bursts (GRBs) are thought to originate at cosmological distances from the most powerful explosions in the universe. If GRBs are not beamed, then the distribution of their number as a function of $\gamma$-ray flux implies that they occur once per $\sim(0.3-40)$ million years per bright galaxy and that they deposit $\gtrsim 10^{53}$ ergs into their surrounding interstellar medium. The blast wave generated by a GRB explosion would be washed out by interstellar turbulence only after tens of millions of years when it finally slows down to a velocity of $\sim 10 \mathrm{~km} \mathrm{~s}^{-1}$. This rather long lifetime implies that there could be up to several tens of active GRB remnants in each galaxy at any given time. For many years, radio observations have revealed the enigmatic presence of expanding neutralhydrogen ( $\mathrm{H}$ I) supershells of near-kiloparsec radius in the Milky Way and in other nearby galaxies. The properties of some supershells cannot be easily explained in terms of conventional sources such as stellar winds or supernova explosions. However, the inferred energy and frequency of the explosions required to produce most of the observed supershells agree with the above GRB parameters. More careful observations and analysis might reveal which fraction of these supershells are GRB remnants. We show that if this link is established, the data on $\mathrm{H}$ I supershells can be used to constrain the energy output, the rate per galaxy, the beaming factor, and the environment of GRB sources in the universe.
\end{abstract}

Subject headings: cosmology: theory — gamma rays: bursts — ISM: bubbles

\section{INTRODUCTION}

By now there is substantial evidence that gamma-ray bursts (GRBs) originate at cosmological distances (Metzger et al. 1997) from unusually powerful explosions (Kulkarni et al. 1997). Ignoring beaming, the distribution of the number of GRBs as a function of $\gamma$-ray flux implies that they occur once per $\sim(0.3-40)$ million years per bright galaxy and that they deposit $\gtrsim 10^{53} \mathrm{ergs}$ into their surrounding interstellar medium (Wijers et al. 1998).

The detection of afterglow emission extending over months after a GRB event (Galama et al. 1998) is most naturally interpreted in terms of delayed synchrotron emission from the relativistic blast wave produced by the GRB explosion in a surrounding medium (see, e.g., Mészáros \& Rees 1997; Waxman 1997b). The inferred density of the ambient gas, $\sim 1 \mathrm{~cm}^{-3}$, is comparable to that of the interstellar medium in spiral galaxies (Waxman 1997b). In such a medium, the expanding blast wave will weaken sufficiently to be washed out by interstellar turbulence or by moving interstellar clouds only after it has slowed down to a velocity of $\sim 10 \mathrm{~km} \mathrm{~s}^{-1}$. For a uniform medium of density $n_{1} \mathrm{~cm}^{-3}$, the late phase of the blast wave evolution would result (Chevalier 1974) in a cool thin H I shell expanding at a velocity $V_{\mathrm{sh}}=4.5 \mathrm{~km} \mathrm{~s}^{-1} E_{54}^{0.71} n_{1}^{-0.80} R_{\mathrm{kpc}}^{-2.2}$, where $E_{54}$ is the initial energy deposited into the gas in units of $10^{54}$ ergs and $R_{\mathrm{kpc}}$ is the shell radius in kiloparsecs. The material is expected to decelerate down to $V_{\mathrm{sh}}=10 \mathrm{~km} \mathrm{~s}^{-1}$ at a radius $R_{\mathrm{kpc}}=0.7 E_{54}^{0.32} n_{1}^{-0.36}$ after a time of $t=\left(0.31 R / V_{\mathrm{sh}}\right)=$ $21 E_{54}^{0.32} n_{1}^{-0.36}$ million years. Since this radius is somewhat larger than the scale height of a galactic disk, the expanding shell might blow a hole in the disk and stretch along the direction of decreasing density (Kompaneets 1960; Chevalier \& Gardner 1974). Moreover, as the remnant lifetime is comparable to a galactic rotation period, its shape could be distorted somewhat by the galactic shear (Tenorio-Tagle \& Bodenheimer 1988).

The rate of GRBs per comoving volume in the universe can be estimated from their number-flux distribution after making an assumption about the redshift evolution of their luminosity function. The simplest model assumes that GRBs are standard candles and allows only for evolution of their rate. If GRBs are further assumed to be a completely nonevolving population, then their rate should be as high as once per (0.3-1) million years per $L_{\star}$ galaxy; on the other hand, if their rate is assumed to be proportional to the cosmic star formation rate, then they should occur once per $\sim 40$ million years per $L_{\star}$ galaxy (Wijers et al. 1998). Since the lifetime of a GRB remnant is several tens of millions of years, we expect to find one to several tens of remnants per galaxy. The inferred energy that a GRB source deposits into the surrounding gas depends on the assumed efficiency, $\epsilon$, for converting this energy into the observed $\gamma$-ray radiation. In the no-evolution model one finds (Wijers et al. 1998) $E_{54} \sim 0.4 \times(\epsilon / 1 \%)^{-1}$, where the typical BATSE source redshift is at $\langle z\rangle \sim 0.8$, while for the stellar evolutionary model (Wijers et al. 1998), $E_{54} \sim 10 \times(\epsilon / 1 \%)^{-1}$ ergs and $\langle z\rangle \sim 2.6$. If GRB sources are beamed and illuminate only a fraction $f_{b}$ of their sky in $\gamma$-rays, then their rate should be higher by a factor $f_{b}^{-1}$ and their energy output should be lower by a factor $f_{b}$. Existing observational data are degenerate to the value of $f_{b}$ (although the degeneracy could be removed by searching for optically selected afterglows with no GRB counterparts; Rhoads 1997). A direct count of the number of GRB remnants per galaxy in the local universe could break this degeneracy and determine the values of $f_{b}$ and $\epsilon$ from the production rate and explosion energy of these remnants. Since almost all GRBs show X-ray afterglow emission, which signals the existence of an external medium (while optical emission could sometimes be suppressed as a result of dust obscuration), interstellar GRB remnants are likely to form in a dominant fraction of all GRB events.

\section{H I SUPERSHELLS}

For several decades, $21 \mathrm{~cm}$ surveys of spiral galaxies have revealed the puzzling existence of expanding giant $\mathrm{H}$ i supershells (see, e.g., Tenorio-Tagle \& Bodenheimer 1988). These nearly spherical structures are deficient of interstellar matter in 
their interiors and have high $\mathrm{H}$ I density at their boundaries that expand at velocities of several tens of $\mathrm{km} \mathrm{s}^{-1}$. The radii of these shells are much larger than those of ordinary supernova remnants and often exceed $\sim 1 \mathrm{kpc}$. By classification (Heiles 1979), they possess inferred kinetic energies of $\gtrsim 3 \times 10^{52}$ ergs, and their estimated ages are in the range of $10^{6}-10^{8} \mathrm{yr}$. The Milky Way galaxy contains probably several tens of supershells (Heiles 1979; Heiles, Reach, \& Koo 1996), and in one case the estimated kinetic energy is as high as $\sim 10^{54}$ ergs. A similar network of supershells, which clearly result from localized depositions of energy into the interstellar medium, is observed in other nearby galaxies. The observed number of supershells is consistent with a production rate of about one per few million years per galaxy. All these characteristics are close to the expected properties of GRB remnants. Nevertheless, the existing literature regards the energy source of these supershells as an unsettled issue and explores other mechanisms for their production-all of which have problems in some specific examples (only Blinnikov \& Postnov 1998 briefly mention the possibility of a connection with GRBs).

Smaller shells of radii $\sim 200-400 \mathrm{pc}$ and energies $\leqslant 3 \times$ $10^{52} \mathrm{ergs}$ are often explained as a consequence of the collective action of stellar winds and supernova explosions originating from OB star associations (McCray \& Kafatos 1987; Shull \& Saken 1995). The activity in an OB association creates a bubble in the interstellar medium that is filled with hot gas. The excess pressure inside the bubble gives kinetic energy to the ambient gas, which collects on a shell around the bubble. After the shell has expanded to a size comparable to the scale height of the galactic disk, its upper part accelerates and becomes RayleighTaylor unstable. Subsequently, a phenomenon called "blowout" might occur, whereby gas escapes from the interior of the bubble into the halo of the galaxy (Igumentschev, Shuston, \& Tutukov 1990). The shell then fragments, and its surviving filamentary pieces are sometimes referred to as "galactic worms." This stellar model could satisfy the energy requirements for shells of modest energies. However, for giant shells with larger kinetic energies this explanation requires extreme and often implausible assumptions. For the Milky Way supershells, the stringent constraints placed by both the number of massive stars present in $\mathrm{OB}$ associations and by the detailed structure of the Galactic disk seem to rule out such associations as the appropriate sources of energy (Tenorio-Tagle \& Bodenheimer 1988). In other galaxies that cannot be imaged at the same level of detail, one could postulate more populous OB associations and still invoke the same mechanism, but there are known cases in which even the minimal assumptions appear very extreme. Two notable examples are the giant $\mathrm{H}$ I supershells discovered (Rand \& van der Hulst 1993) in NGC 4631, with radii $R_{1}=0.9 \mathrm{kpc}$ and $R_{2}=1.5 \mathrm{kpc}$, and kinetic energies $E_{1}=(6-10) \times 10^{53} \mathrm{ergs}$ and $E_{2}=(2-5) \times 10^{54} \mathrm{ergs}$, respectively. If these shells were to be formed by multiple stellar winds and supernova explosions, the required $\mathrm{OB}$ associations would need to have $(4-10) \times 10^{3}$ OB stars for shell 1 and $(1-3.5) \times 10^{4}$ stars for shell 2 . But associations with $\gtrsim 10^{4} \mathrm{OB}$ stars would be unprecedented for a galactic disk. The H II region formed by an association of $\sim 10^{4} \mathrm{OB}$ stars would have an $\mathrm{H} \alpha$ luminosity of $6 \times 10^{40} \mathrm{ergs} \mathrm{s}^{-1}$ and rank as the brightest among thousands of $\mathrm{H}$ II regions in nearby galaxies (Kennicutt, Edgar, \& Hodge 1989). However, such large associations are not impossible (Williams \& McKee 1997).

An alternative model suggests that impacts of small companions or high-velocity gas clouds on the galactic disk have formed the observed shells (Tenorio-Tagle 1981). However, it is unclear how such collisions could account for the near-complete ringlike appearance of the supershell boundaries (Rand \& van der Hulst 1993). Moreover, the supershell sizes and kinetic energies imply that the collisions must have been far more energetic than those thought to occur between high-velocity clouds and the Galactic disk, where the available energies are $\lesssim 2 \times 10^{52}$ ergs. A search for independent evidence that massive objects might have passed through the disk of NGC 4631 has failed (Rand \& van der Hulst 1993).

The giant supershells of NGC 4631 are two examples out of several (Rhode, Salzer, \& Westpfahl 1997) in which a more satisfying explanation for the nature of the energy source is needed. We suggest that some supershells are GRB remnants. In fact, given the number-flux distribution of GRBs and the interpretation of GRB afterglows as emission from an interstellar blast wave, it is unavoidable to find some active remnants with the properties of the largest supershells in galaxies. The GRB-produced supershells might, however, be a minority population. Unfortunately, the current data on the statistics and properties of supershells are sketchy and inconclusive and may not be sufficient for drawing any firm quantitative conclusions. In the next section we will attempt to illustrate the potential implications that might result from better $\mathrm{H}$ I data on the supershell properties and better optical data on the absence of conventional energy sources to explain them. For this purpose alone, we will tentatively adopt rough numbers which were quoted in the literature (Heiles 1979; Tenorio-Tagle \& Bodenheimer 1988) and assume that most of the observed supershells are produced by GRBs.

\section{IMPLICATIONS FOR GRBS}

From the size and velocity of the observed supershells we estimate that a typical GRB event would have to release $\sim 10^{54}$ ergs (and in rare cases even 10 times more energy), assuming that a few to 10 percent of the initial energy ends up in the final kinetic energy of the supershell and the rest is radiated away (Chevalier 1974). The inferred output energy is close to the rest mass energy of a solar mass object, and might possibly arise from the collapse of a massive star to a black hole (Woosley 1993; Paczyńsky 1998). The continuous distribution of explosion energies for shells of different sizes in the interstellar medium, ranging from the typical output of a single supernova $\sim 10^{51}$ ergs and up to $\sim 10^{55}$ ergs, would be natural in this interpretation. The upper end of this energy range is comparable to the spin energy of a maximally rotating $\sim 10 M_{\odot}$ black hole that could be tapped magnetically through outgoing jets and produce the observed GRB events (Paczyńsky 1998). On the other hand, it would be more difficult to reach this extreme energy output through the coalescence of a binary neutron-star system. Detailed theoretical modeling of the observed afterglow emission in some GRBs (Waxman 1997b) implies that only $\lesssim 10^{53}$ ergs are being carried by highly relativistic matter in the first few months of the explosion. Our energy estimate suggests that 10 times more energy might be outflowing with nonrelativistic matter that eventually catches up and energizes the outgoing blast wave on a longer timescale, after the shock has decelerated to a sufficiently low velocity. The enhancement factor might relate naturally to the value of $f_{b}^{-1}$ if the relativistic material is beamed. The process that reenergizes the shock should leave a clear signature on the late-time evolution of the afterglow light curve (Paczyńsky 1998).

Typically, there are of order 10 supershells detected per galaxy (Heiles 1979; Thilker, Braun, \& Walterbros 1998), and 
their estimated lifetime is a few tens of millions of years. The inferred values for the explosion energy $\left(\propto f_{b} / \epsilon\right)$ and production rate $\left(\propto f_{b}^{-1}\right)$ of supershells imply a radiative efficiency of $\epsilon \sim$ $0.4 \%$ and no beaming in the no-evolution model for the GRB population, while they require $\epsilon \sim 1 \%$ and a beaming fraction of $f_{b} \sim 10 \%$ for the evolutionary model where the GRB rate follows the cosmic star formation history. Both models predict roughly the same (reasonable) value for $\epsilon$, and rule out strong beaming with $f_{b} \ll 10 \%$ because it implies a much larger population of $\mathrm{H}$ I supershells than observed. This latter constraint is robust since the GRB rate increases as $f_{b}^{-1}$, while the supershell radius declines only as $f_{b}^{0.32}$. We note that even if the energy is injected impulsively along a pair of relativistically beamed jets, the nonrelativistic expansion of the eventual $\mathrm{H} \mathrm{I}$ supershell would be close to spherical.

The identification of the centers of the youngest supershells can be used to reveal the probable location of GRB explosions. This could test models that associate GRBs with star-forming regions (Woosley 1993; Paczyńsky 1998) or, alternatively, rule out models that relate them to active galactic nuclei or to binaries involving a neutron star-which should be kicked away from star-forming regions by the time they coalesce (Tutukov \& Yungelson 1994). It would also be natural to search for unusual emission that might be associated with a potential remnant object inside these supershells, although any such object is likely to get kick velocities far in excess of the final supershell velocity and hence exit the supershell boundary. In general, it should be easier to separate GRB remnants from supernova remnants during the early (e.g., adiabatic) phase of their ev- olution. As it is highly unlikely that the separate explosions of $\sim 10^{3}$ supernovae would be synchronized to within a period shorter than a million years, a young GRB remnant would contain far more kinetic (or thermal) energy than OB associations might account for.

The rate of energy injection by GRBs per unit volume in the current universe can be obtained by multiplying their energy output by their frequency and by the density of $L_{\star}$ galaxies, yielding $\sim\left(10^{54} \mathrm{ergs}\right)\left(2 \times 10^{-3} \mathrm{Mpc}^{-3}\right) /\left(2 \times 10^{6} \mathrm{yr}\right)=$ $10^{45} \mathrm{ergs} \mathrm{Mpc}^{-3} \mathrm{yr}^{-1}$. This estimate is based on the fact that most of the current star formation occurs in massive $L_{\star}$ galaxies. The result appears to be close to the energy injection rate per unit volume of $10^{19-21} \mathrm{eV}$ cosmic rays (Waxman 1997a),

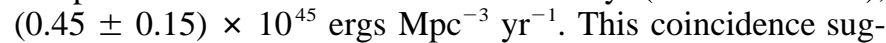
gests that GRBs might provide the acceleration sites for the highest energy cosmic rays (Waxman 1995) and that the accelerated particles possibly approach equipartition with the magnetic and thermal energy densities behind GRB shocks.

The above analysis illustrates the importance of getting better data on the subpopulation of $\mathrm{H}$ I supershells, which cannot be associated with conventional energy sources. We have demonstrated that the detailed properties of this population could determine the basic parameters of GRBs and possibly hint as to their most likely energy source.

We thank John Raymond and Eli Waxman for useful discussions. This work was supported in part by a NASA HST grant, an ATP grant NAG5-3085, and the Harvard Milton fund.

\section{REFERENCES}

Blinnikov, S. I., \& Postnov, K. A. 1998, MNRAS, 293, L29

Chevalier, R. A. 1974, ApJ, 188, 501

Chevalier, R. A., \& Gardner, J. 1974, ApJ, 192, 457

Galama, T. J., et al. 1998, ApJL, in press (preprint astro-ph/9802160)

Heiles, C. 1979, ApJ, 229, 533

Heiles, C., Reach, W. T., \& Koo, B.-C. 1996, ApJ, 466, 191

Igumentschev, I. V., Shuston, B. M., \& Tutukov, A. V. 1990, A\&A, 234, 396

Kennicutt, R. C., Edgar, B. K., \& Hodge, P. W. 1989, ApJ, 337, 761

Kompaneets, A. S. 1960, Sov. Phys. Doklady, 5, 46

Kulkarni, S. R., et al. 1997, Nature, 393, 35

McCray, R., \& Kafatos, M. 1987, ApJ, 317, 190

Mészáros, P., \& Rees, M. J. 1997, ApJ, 476, 232

Metzger, M. R., et al. 1997, Nature, 387, 879

Paczyńsky, B. 1998, ApJ, 494, L45

Rand, R. J., \& van der Hulst, J. M. 1993, AJ, 105, 2098
Rhoads, J. E. 1997, ApJ, 487, L1

Rhode, K. L., Salzer, J. J., \& Westpfahl, D. J. 1997, BAAS, 191, 81.09

Shull, J. M., \& Saken, J. M. 1995, ApJ, 444, 663

Tenorio-Tagle, G. 1981, A\&A, 94, 338

Tenorio-Tagle, G., \& Bodenheimer, P. 1988, ARA\&A, 26, 145

Thilker, D. A. Braun, R., \& Walterbros, R. A. M. 1998, A\&A, 332, 429

Tutukov, A. V., \& Yungelson, L. R. 1994, MNRAS, 268, 871

Waxman, E. 1995, Phys. Rev. Lett., 75, 386

1997a, ApJ, 452, L1 1997b, ApJ, 489, L33

Wijers, R. A. M. J., Bloom, J. S., Bagla, J. S., \& Natarajan, P. 1998, MNRAS, 294, L13

Williams, J. P., \& McKee, C. F. 1997, ApJ, 476, 166

Woosley, S. E. 1993, ApJ, 405, 273 